\title{
Aortic Valve Calcification Is Associated with Future Cognitive Impairment
}

\author{
Hojune E. Chunga,b, Jessica Chen ${ }^{\mathrm{b}}$, Dhairyasheel Ghosalkar ${ }^{\mathrm{b}}$, Jared L. Christensen ${ }^{\mathrm{a}, \mathrm{b}}$, Alice J. Chu ${ }^{\mathrm{b}}$, \\ Chris S. Mantsounga ${ }^{\mathrm{a}, \mathrm{b}}$, Jade Neverson ${ }^{\mathrm{a}, \mathrm{b}}$, Cullen Soares $^{\mathrm{b}}$, Nishant R. Shah ${ }^{\mathrm{a}, \mathrm{b}}$, Wen-Chih Wu ${ }^{\mathrm{a}, \mathrm{b}}$, \\ Gaurav Choudhary ${ }^{\mathrm{a}, \mathrm{b}}$ and Alan R. Morrison ${ }^{\mathrm{a}, \mathrm{b}, *}$ \\ ${ }^{a}$ Department of Internal Medicine (Section of Cardiovascular Medicine), Providence VA Medical Center, \\ Providence, RI, USA \\ ${ }^{\mathrm{b}}$ Department of Internal Medicine (Section of Cardiovascular Medicine), Alpert Medical School at Brown \\ University, Providence, RI, USA
}

Accepted 9 March 2021

Pre-press 30 March 2021

\begin{abstract}
.
Background: While an association between atherosclerosis and dementia has been identified, few studies have assessed the longitudinal relationship between aortic valve calcification (AVC) and cognitive impairment (CI).

Objective: We sought to determine whether AVC derived from lung cancer screening CT (LCSCT) was associated with CI in a moderate-to-high atherosclerotic risk cohort.

Methods: This was a single site, retrospective analysis of 1401 U.S. veterans (65 years [IQI: 61, 68] years; 97\% male) who underwent quantification of AVC from LCSCT indicated for smoking history. The primary outcome was new diagnosis of CI identified by objective testing (Mini-Mental Status Exam or Montreal Cognitive Assessment) or by ICD coding. Time-to-event analysis was carried out using AVC as a continuous variable.

Results: Over 5 years, 110 patients (8\%) were diagnosed with CI. AVC was associated with new diagnosis of CI using 3 Models for adjustment: 1) age (HR: 1.104; CI: 1.023-1.191; $p=0.011)$; 2) Model 1 plus hypertension, hyperlipidemia, diabetes, CKD stage 3 or higher (glomerular filtration rate $<60 \mathrm{~mL} / \mathrm{min}$ ) and CAD (HR: 1.097; CI: 1.014-1.186; $p=0.020$ ); and 3) Model 2 plus CVA (HR: 1.094; CI: 1.011-1.182; $p=0.024)$. Sensitivity analysis demonstrated that the association between AVC and new diagnosis of CI remained significant upon exclusion of severe AVC (HR: 1.100 [1.013-1.194]; $p=0.023$ ). Subgroup analysis demonstrated that this association remained significant when including education in the multivariate analysis (HR: 1.127 [1.030-1.233]; $p=0.009$ ).

Conclusion: This is the first study demonstrating that among mostly male individuals who underwent LCSCT, quantified aortic valve calcification is associated with new diagnosis of CI.
\end{abstract}

Keywords: Atherosclerosis, calcific aortic valve disease, computed tomography, dementia

\section{INTRODUCTION}

Cognitive impairment $(\mathrm{CI})$ is a growing problem in an increasingly aging population and is expected

\footnotetext{
*Correspondence to: Alan R. Morrison, MD, PhD, Providence VA Medical Center, Research (151), 830 Chalkstone Avenue, Providence, RI 02908, USA. E-mail: alan_morrison@brown.edu.
}

to affect approximately 13.8 million Americans by 2050 [1]. The classification of CI into mild cognitive impairment versus dementia is dependent on identifying decreased mental abilities and impairment of daily functioning [2]. Early identification of patients at risk for CI may be of value to clinicians as studies have shown that the conversion rate of mild cognitive impairment to dementia can be as much as $30 \%$ over 
5 years [3]. However, few biomarkers exist that reliably predict disease incidence at early or preclinical stages much less risk of progression [4].

Inflammation and atherosclerotic vasculopathy, including microangiopathy, have been strongly associated with the neurodegenerative changes associated with CI [5, 6]. In addition, onset of CI is strongly influenced by cerebrovascular accident (CVA), and by the number of CVA events, with CI occurring in $10 \%$ of patients after an initial CVA and 30\% after recurrent CVA [7].

Our group recently found aortic valve calcification (AVC) to be independently associated with increased atherosclerotic events, including all-cause mortality, nonfatal myocardial infarction (MI), and nonfatal CVA, despite a very weak correlation between AVC and coronary calcification [8]. Other groups have demonstrated the presence of AVC to be associated with covert brain infarcts identified on MRI [9]. Moreover, cerebral white matter abnormalities, including those attributable to CVA, have been associated with cognitive decline and dementia $[10,11]$

While an association between atherosclerotic vascular disease and CI has been identified, few studies have assessed the longitudinal relationship between AVC and CI [12]. Here we sought to determine the prognostic value of AVC derived from low dose, lung cancer screening computed tomography (LCSCT) for $\mathrm{CI}$ in a moderate-to-high atherosclerotic risk cohort.

\section{MATERIALS AND METHODS}

This study was approved by the Providence Veteran's Affairs (VA) Medical Center Institutional Review Board and complies with the Declaration of Helsinki, and all patient data were handled in compliance with the Health Insurance Portability and Accountability Act (HIPAA) regulations. All patient records were de-identified and analyzed anonymously. Through the Providence VA Medical Center and the corresponding office of the Associate Chief of Staff for Research, all data, analytical methods, and study materials are available upon request to other researchers, who meet the Institutional Review Board criteria for access to VA confidential research data, for purposes of reproducing the results or replicating the study.

This was a single-center, retrospective analysis of 1654 U.S. veterans who underwent U.S. Preventive Services Task Force guideline-recommended (i.e., 30 -pack-year tobacco smoking history, 55 to 80 years of age, active or quit $<5$ years previously) LCSCT between October 1, 2013, and July 31, 2014 [13]. A total of 253 patients were excluded from the study due to diagnosis of lung cancer on the LCSCT, prior aortic valve replacement and prior diagnosis of CI resulting in 1401 patients for all subsequent analyses. Prior aortic valve replacement and CI were defined as occurring prior to the date of the LCSCT.

CT examinations were not electrocardiographically (ECG)-gated and were performed with a 128-slice CT scanner (Siemens Healthcare, Erlangen, Germany); $128 \times 0.6 \mathrm{~mm}$ collimation, 0.5 -s rotation, pitch of $0.84,380-\mathrm{mm}$ field of view, $512 \times 512$ pixel matrix, tube voltage of $120 \mathrm{kV}$ and tube current of $40 \mathrm{~mA}$, image reconstruction thickness of $1.00-1.25 \mathrm{~mm}$, and a minimum area required to identify calcium was $0.55 \mathrm{~mm}^{2}$. AVC and coronary artery calcification (CAC) scores were quantified using the Agatston method via a semiautomated imaging workstation with readers blinded to patient data $[14,15]$. The kappa for interobserver agreement was 0.91 $(0.85-0.95)$ and $0.92(0.88-0.96)$ for AVC and CAC, respectively. As described elsewhere, there was a strong Pearson correlation between AVC from nonECG-gated and ECG-gated CT acquisition protocols $[8,16]$.

The U.S. Department of Veterans Affairs (VA) electronic medical record (EMR) was searched for patient demographics and cardiovascular risk factors. Coronary artery disease (CAD) was defined as a history of MI, prior revascularization or abnormalities on cardiac testing (exercise treadmill testing, echocardiography, myocardial perfusion, cardiac computed tomography, or coronary angiography). MI was defined as EMR-documented evidence of elevated cardiac troponin values with at least one value above the $99^{\text {th }}$ percentile upper reference limit plus clinical evidence of acute myocardial ischemia, consistent with universal definitions [17]. CVA included nonhemorrhagic, ischemic stroke and transient ischemic attack.

The primary outcome was new diagnosis of CI identified by objective cognitive testing or by ICD coding, using the following diagnosis codes: ICD9:290, 294.11, 331.0, 331.1, 331.2, 331.82, 331.83, 331.9, 438.0, and 780.93; ICD-10:294.8, 294.9, 437.9, 780.93, F00, F01, F02.81, F03, F03.91, F04, F09, G30, G31, G31.84, I67.9, I69.898, I69.918, R41, R41.81, R41.840, and R41.9 (Supplementary Table 1). The ICD-9 and ICD-10 diagnosis codes for CI have been shown to have a sensitivity of $32.3 \%$ and $66.9 \%$ and a specificity of $100 \%$ and 
Table 1

Baseline demographics and clinical characteristics of overall population

\begin{tabular}{lc}
\hline & $\begin{array}{c}\text { Overall Population } \\
(n=1,401)\end{array}$ \\
\hline Age, y, median (IQI) & $65(61,68)$ \\
BMI, median (IQI) & $28.7(25.4,32.6)$ \\
Male, $\mathrm{n}(\%)$ & $1353(96.6)$ \\
Caucasian, $\mathrm{n}(\%)$ & $1321(94.5)$ \\
DM, $\mathrm{n}(\%)$ & $386(27.6)$ \\
Hypertension, $\mathrm{n}(\%)$ & $857(61.2)$ \\
Hyperlipidemia, $\mathrm{n}(\%)$ & $1,047(74.7)$ \\
Total Cholesterol, median (IQI) & $177(154,204)$ \\
HDL Cholesterol, median (IQI) & $43(36,52)$ \\
Statin Use, $\mathrm{n}(\%)$ & $825(58.9)$ \\
Current Smoker, $\mathrm{n}(\%)$ & $756(54.0)$ \\
Family History of Early CAD, n (\%) & $166(11.9)$ \\
CAD, $\mathrm{n}(\%)$ & $325(23.2)$ \\
Prior MI, $\mathrm{n}(\%)$ & $143(10.2)$ \\
Prior CABG, $\mathrm{n}(\%)$ & $59(4.2)$ \\
Prior CVA, $\mathrm{n}(\%)$ & $80(5.7)$ \\
CKD, $\mathrm{n}(\%)$ & $197(14.1)$ \\
GFR, median (IQI) & $79.9(69.3,92.4)$ \\
ASCVD Risk Score, median (IQI) & $18.6(12.4,28.0)$ \\
CACS, median (IQI) & $528(105,1512)$ \\
AVC, median (IQI) & $60(0,338)$ \\
Diagnosis of CI, n (\%) & $110(7.9)$ \\
\hline AVC, aotic valve
\end{tabular}

AVC, aortic valve calcium score; ASCVD, atherosclerotic cardiovascular disease; BMI, body mass index; $\mathrm{CABG}$, coronary artery bypass graft surgery; $\mathrm{CACS}$, coronary artery calcium score; $\mathrm{CAD}$, coronary artery disease; CKD, chronic kidney disease; CVA, cerebrovascular accident; DM, diabetes mellitus; HDL, high-density lipoprotein; GFR, glomerular filtration rate; CI, cognitive impairment; MI, myocardial infarction.

$99.8 \%$, respectively [18]. Objective cognitive testing included patients evaluated by either Mini-Mental Status Exam (MMSE) or Montreal Cognitive Assessment (MoCA). The MMSE and MoCA items have been described in detail elsewhere and the utilized cut-off scores for CI by MMSE and MoCA are less than 26 and less than 24, respectively [19, 20]. Evaluations were conducted by the Mental Health and Behavioral Science Services (MHBSS) at the VA by trained behavioral mental health providers. Scores were not adjusted for age or education. Similar to prior studies, the MHBSS documentation detailed the highest educational attainment in the following categories: $8^{\text {th }}$ grade and below, some High School, High School Diploma or General Education Development (GED), some College or Associate's Degree, Bachelors and Post-Bachelor degree [21]. The highest educational attainment was recorded as a categorical variable. Adjudicators of clinical outcomes were blinded to AVC and CAC and vice versa.

Results are presented as mean (standard error) for continuous variables with normal distribution, median (interquartile range) for continuous variables without normal distribution, and number (percentage) for categorical data. The Shapiro-Wilk test for normality was used to test for normal distribution of age, body mass index (BMI), hyperlipidemia, atherosclerotic cardiovascular disease (ASCVD), CAC and AVC.

The relationship between AVC as a continuous variable and primary outcomes was determined using Cox regression analysis. AVC was treated as continuous variable, using base-10 log transformation of $\mathrm{AVC}+1(\log [\mathrm{AVC}+1])$ to account for a right-skewed distribution. The statistical analysis included 3 separate models: Model 1 adjusting for age; Model 2 adjusting for age, hypertension, hyperlipidemia, diabetes mellitus, chronic kidney disease (CKD) stage 3 or higher (glomerular filtration rate $<60 \mathrm{~mL} / \mathrm{min}$ ) and CAD; and Model 3 adjusting for age, hypertension, hyperlipidemia, diabetes mellitus, CKD stage 3 or higher (glomerular filtration rate $<60 \mathrm{~mL} / \mathrm{min}$ ), $\mathrm{CAD}$ and prior CVA. A sensitivity analysis was performed by excluding those patients with severe AVC, as defined by $\geq 1,274 \mathrm{AU}$ in women and $\geq 2,065 \mathrm{AU}$ in men [22, 23]. A subgroup analysis was performed on a subset of patients for whom highest education attainment was documented in the VA EMR, allowing for inclusion of highest education attainment as a categorical in the multivariate adjustment.

All statistical analyses were conducted with the use of Stata/SE (version 15.1; StataCorp LLC, College Station, TX) or Prism (version 7; GraphPad Software Inc, La Jolla, CA). For all statistical analyses, a $p$ value less than 0.05 was considered significant.

\section{RESULTS}

We evaluated a total of 1401 patients who underwent clinically indicated low dose lung cancer screening CT at Providence VA Medical between October 1, 2013 and July 31, 2014 (Table 1). Indicative of the northeast U.S. veteran population, the median age of this mostly Caucasian (94\%), male $(97 \%)$ population was 65 years (interquartile interval [IQI]: 61, 68 years). The median ASCVD risk score was $18.6 \%$ (IQI: $12.4 \%, 28.0 \%$ ). Sixty-one percent of patients had hypertension, $75 \%$ had dyslipidemia, $28 \%$ had diabetes, $14 \%$ had CKD, $6 \%$ had prior CVA or TIA, and $23 \%$ had CAD. The overall median CAC was 528 AU (IQI: 105, 1,512). The overall median AVC was 60 AU (IQI: 0, 338). 
Table 2

Relationship between aortic valve calcification and cognitive impairment

\begin{tabular}{lccc}
\hline & \multicolumn{3}{c}{ Cognitive Impairment } \\
\cline { 2 - 4 } Model & HR & $(95 \%$ CI $)$ & $p$ \\
\hline 1 & 1.104 & $1.023-1.191$ & 0.011 \\
2 & 1.097 & $1.014-1.186$ & 0.020 \\
3 & 1.094 & $1.011-1.182$ & 0.024 \\
\hline
\end{tabular}

Covariate adjustment by model: Model 1=age. Model 2=age, hypertension, hyperlipidemia, CKD, diabetes, and CAD. Model $3=$ age, hypertension, hyperlipidemia, CKD, diabetes, CAD, and prior CVA.

Over 5 person-years of follow-up, 110 patients (7.9\%) were newly diagnosed with CI. Of these 110 patients, 74 patients were diagnosed by objective testing with MoCA (71 patients) and MMSE (3 patients). Thirty-six additional patients were identified by ICD coding. By Cox regression, AVC was associated with a new diagnosis of CI (HR: 1.151; 95\% CI: $1.071-1.236 ; p<0.001)$. In Model 1, the association between AVC and new diagnosis of CI remained significant after adjustment for age (HR: 1.104; CI: 1.023-1.191; $p=0.011$; Table 2). In Model 2 , the association between AVC and new diagnosis of CI remained significant after adjustment for age, hypertension, hyperlipidemia, diabetes, CKD stage 3 or higher (glomerular filtration rate $<60 \mathrm{~mL} / \mathrm{min}$ ) and CAD (HR: 1.097; CI: 1.014-1.186; $p=0.020$; Table 2). In Model 3, the association between $\mathrm{AVC}$ and new diagnosis of CI remained significant after multivariate adjustment for age, hypertension, hyperlipidemia, diabetes, CKD stage 3 or higher (glomerular filtration rate $<60 \mathrm{~mL} / \mathrm{min}$ ), $\mathrm{CAD}$ and prior CVA (HR: 1.094; CI: 1.011-1.182; $p=0.024$; Table 2).

We next performed a sensitivity analysis excluding 46 patients who met criteria for severe AVC by previously established CT criteria $(\geq 1,274$ AU in women and $\geq 2,065 \mathrm{AU}$ in men) to assess the impact of hemodynamics on the primary outcome [22, 23]. Over 5 person-years of follow-up, 104 patients $(7.6 \%)$ were newly diagnosed with $\mathrm{CI}$ in this nonsevere AVC subset. In this subset analysis, AVC was associated with a new diagnosis of CI by Cox regression (HR: 1.151; 95\% CI: 1.067-1.242; $p<0.001)$. Model 1 demonstrated that the association of AVC and new diagnosis of CI remained significant after adjustment for age (HR: 1.109; CI: 1.024-1.202; $p=0.011$; Table 3). Model 2 demonstrated that the association of AVC and new diagnosis of CI remained significant after adjustment for age, hypertension, hyperlipidemia, diabetes, CKD stage
Table 3

Relationship between aortic valve calcification and cognitive impairment in patients with nonsevere AVC

\begin{tabular}{lccc}
\hline & \multicolumn{3}{c}{ Cognitive Impairment } \\
\cline { 2 - 4 } Model & HR & $(95 \%$ CI $)$ & $p$ \\
\hline 1 & 1.109 & $1.024-1.202$ & 0.011 \\
2 & 1.102 & $1.015-1.197$ & 0.020 \\
3 & 1.100 & $1.013-1.194$ & 0.023 \\
\hline
\end{tabular}

Covariate adjustment by model: Model $1=$ age. Model $2=$ age, hypertension, hyperlipidemia, CKD, diabetes, and CAD. Model $3=$ age, hypertension, hyperlipidemia, CKD, diabetes, CAD, and prior CVA.

3 or higher (glomerular filtration rate $<60 \mathrm{~mL} / \mathrm{min}$ ) and CAD (HR: 1.102; CI: 1.015-1.197; $p=0.020$; Table 3). Model 3 demonstrated that the association of AVC and new diagnosis of CI remained significant after adjustment for age, hypertension, hyperlipidemia, diabetes, CKD stage 3 or higher (glomerular filtration rate $<60 \mathrm{~mL} / \mathrm{min}$ ), $\mathrm{CAD}$ and prior CVA (HR: 1.100; CI: 1.013-1.194; $p=0.023$; Table 3).

Of the 1,401 patients, a subset of 803 patients (57.3\%) patients had VA EMR documentation of highest education attainment, allowing for inclusion of educational attainment as a categorical variable in the multivariate adjustment. From this subgroup, 707 patients $(88.04 \%)$ had at least a High School Diploma/GED and 406 patients $(50.56 \%)$ had some form of postsecondary education: 24 patients $(2.99 \%)$ $8^{\text {th }}$ grade and below; 72 patients $(8.97 \%)$ some High School; 301 patients (37.48\%) High School Diploma or GED; 273 patients $(34.00 \%)$ some College or Associate's Degree, 108 patients (13.45\%) Bachelors and 25 patients $(3.11 \%)$ Post-Bachelor degree. Over 5 person-years of follow-up, 87 patients $(10.8 \%)$ were newly diagnosed with $\mathrm{CI}$ in this education attainment subgroup. In the subgroup analysis, AVC was associated with a new diagnosis of $\mathrm{CI}$ by Cox regression (HR: 1.194; 95\% CI: 1.099-1.296; $p<0.001$ ). Model 1 demonstrated that the association of AVC and new diagnosis of CI remained significant after adjustment for age (HR: 1.141; CI: 1.046-1.246; $p=0.003$; Table 4). Model 2 demonstrated that the association of AVC and new diagnosis of CI remained significant after adjustment for age, hypertension, hyperlipidemia, diabetes, CKD stage 3 or higher (glomerular filtration rate $<60 \mathrm{~mL} / \mathrm{min}$ ), $\mathrm{CAD}$, and education (HR: 1.127; CI: 1.030-1.234; $p=0.009$; Table 4). Model 3 demonstrated that the association of AVC and new diagnosis of CI remained significant after adjustment for age, hypertension, hyperlipidemia, diabetes, CKD stage 3 or higher (glomerular filtration rate $<60 \mathrm{~mL} / \mathrm{min}$ ), $\mathrm{CAD}$, prior CVA and 
Table 4

Relationship between aortic valve calcification and cognitive impairment in subset patients with known highest education attainment

\begin{tabular}{lccc}
\hline & \multicolumn{3}{c}{ Cognitive Impairment } \\
\cline { 2 - 4 } Model & HR & $(95 \%$ CI $)$ & $p$ \\
\hline 1 & 1.141 & $1.046-1.246$ & 0.003 \\
2 & 1.127 & $1.030-1.234$ & 0.009 \\
3 & 1.127 & $1.030-1.233$ & 0.009 \\
\hline
\end{tabular}

Covariate adjustment by model: Model $1=$ age. Model $2=$ age, hypertension, hyperlipidemia, CKD, diabetes, CAD, and education. Model 3 = age, hypertension, hyperlipidemia, CKD, diabetes, CAD, education, and prior CVA.

education (HR:1.127; CI: $1.030-1.233 ; p=0.009$; Table 4).

\section{DISCUSSION}

Calcific aortic valve disease (CAVD) shares many common risk factors with atherosclerotic coronary artery disease, including tobacco cigarette smoking [24]. Despite these similarities, HMG-CoA reductase inhibitors (statins), which have had tremendous success at reducing atherosclerotic disease events, do not appear to impact the natural progression of CAVD, suggesting some biological differences between CAVD and atherosclerosis [24-27].

Aortic valve calcification may be a prognostic indicator for atherosclerotic processes affecting various vascular beds, including those associated with the central nervous system [8]; however, the one prior study assessing the relationship between AVC and CI did not demonstrate a significant association [12]. Here we identify that AVC, as a continuous variable, is associated with $\mathrm{CI}$ in patients with an extensive smoking history. The difference between the previous study and our current findings may largely reflect differences in the study cohorts. Overall, the patient cohort in the prior study was at a lower risk from atherosclerotic cardiovascular disease than our cohort which was at moderate-to-high risk of cardiovascular disease as reflected by the increased vascular calcification and the increased incidence of events [28]. Further comparison demonstrated a higher prevalence of cardiovascular risk factors in our cohort relative to the prior study cohort: hypertension (61.2\% versus $40.3 \%)$, hyperlipidemia (74.7\% versus $24.5 \%$ ), diabetes ( $27.6 \%$ versus $12.6 \%$ ), and CAD ( $23.2 \%$ versus $8.1 \%$ ). Moreover, by study design, our cohort had such a significant smoking history as to be eligible for LCSCT, and our patient cohort had a significantly higher percentage of current smokers at
$54 \%$ (versus $12.6 \%$ in the prior study). When examining outcomes, $67 \%$ of patients in the prior study had an AVC score of 0 and $33 \%$ of patients had a nonzero AVC scores, whereas in our study, $36 \%$ patients had an AVC score of 0 and $64 \%$ of patients had a nonzero AVC score. This suggests that the prior study may have been somewhat underpowered due to the predominance of patients with AVC score of zero.

This study was limited by selection biases inherent to the study design. This particular population of northeast U.S. veterans consisted mostly of older, white men with significant smoking histories. While ICD-coding can be highly specific, the sensitivity is relatively low, and therefore, number of new diagnoses of CI may be underestimated. Overall, events may also be underestimated because of limitations inherent in extracting data or because care outside the Department of Veterans Affairs was not fully captured. After the multivariate analysis, there was attenuation after adjustment, raising the potential question of residual confounding. Using E-value analysis [29], the observed hazard ratio in Model 3 of 1.094 could be explained away by an unmeasured confounder that was associated with both the treatment and the outcome by a hazard ratio of 1.41 -fold each, above and beyond the measured confounders, but weaker confounding could not do so; the confidence interval could be moved to include the null by an unmeasured confounder that was associated with both the treatment and the outcome by a hazard ratio of 1.12-fold each, above and beyond the measured confounders, but weaker confounding could not do so. As a retrospective study, information regarding the brain parenchymal status with regard to pathology or imaging were not available.

Hemodynamic impairment from severe AVC, and hence severe physiologic aortic valve stenosis, may also play a role in driving outcomes; however, sensitivity analyses excluding all patients with severe AVC $(\geq 1,274$ AU in women and $\geq 2,065$ AU in men) appeared to have no impact on the association between AVC and new diagnosis of CI, suggesting that the incidence of $\mathrm{CI}$ in our study population was less likely to be driven by hemodynamic changes associated with severe valve disease. CI objective testing scores were not adjusted for age or education; however, according to a recent study of the U.S. veteran population, $90.1 \%$ of veterans held at least a high school diploma or equivalent and $58.8 \%$ had some form of postsecondary education [30]. Our subset analysis demonstrated a comparable highest education attainment with $88.04 \%$ of veterans 
holding at least a high school diploma and 50.56\% of veterans with some form of postsecondary education. Moreover, in our subgroup analysis, inclusion of education in the multivariate adjustments did not attenuate the association between AVC and new diagnosis of CI, suggesting that educational status was not the key driver of outcomes in our cohort. To address these limitations more completely, further studies are needed, including larger prospective multicenter studies in a more diverse patient population.

In conclusion, scoring AVC derived from LCSCT may be helpful at identifying risk of future $\mathrm{CI}$ in patient populations with CAVD and a high risk of atherosclerotic vascular disease. Previous studies have shown that both CAC and AVC derived from non-ECG-gated CT have strong agreement with CAC and AVC derived from ECG-gated CT $[8,16]$. Thus, reporting AVC from LCSCT may help physicians identify patients who would benefit from more aggressive cardiovascular care, including early forms of vascular dementia. Additional prospective studies are required to further assess the mechanistic relationship between atherosclerotic or thrombotic events and CI in patients with high AVC in order to determine whether this population would benefit from more aggressive primary and secondary strategies involving lipid/inflammation-based cardiovascular treatment or even antiplatelet/anticoagulant therapies.

\section{ACKNOWLEDGMENTS}

Research reported in this publication was supported by a Research Project Grant NIH NHLBI R01HL139795 (A.R.M.), Research Project Grant NIH NHLBI R01HL148727 (G.C.) and an Institutional Development Award (IDeA) from NIH NIGMS P20GM103652 (A.R.M.) This work was also supported by Career Development Award Number 7IK2BX002527 from the United States Department of Veterans Affairs Biomedical Laboratory Research and Development Program (A.R.M.), VA VHA CSR\&D 1I01CX001892 (G.C.), and Agency for Healthcare Research and Quality Award 5K12HS022998 (N.R.S.). The views expressed in this article are those of the authors and do not necessarily reflect the position or policy of the Department of Veterans Affairs or the United States government. This work was also supported by a Lifespan CVI Pilot Grant for Faculty (A.R.M.) and a Lifespan CVI Pilot Grant for Fellows (H.E.C.). This work was also supported in part by NIH NHLBI T35 HL094308 (A.C.), by NIH NHLBI R25 HL088992 (J.N.), National Institute of General Medical Sciences award U54GM115677 (N.R.S.), and the Providence VA Medical Service (W.W.).

\section{CONFLICT OF INTEREST}

The authors have no conflict of interest to report.

\section{SUPPLEMENTARY MATERIAL}

The supplementary material is available in the electronic version of this article: https://dx.doi.org/ 10.3233/ADR-200253.

\section{REFERENCES}

[1] Hebert LE, Weuve J, Scherr PA, Evans DA (2013) Alzheimer disease in the United States (2010-2050) estimated using the 2010 census. Neurology 80, 1778-1783.

[2] Knopman DS, Petersen RC (2014) Mild cognitive impairment and mild dementia: A clinical perspective. Mayo Clinic Proc 89, 1452-1459.

[3] Ward A, Tardiff S, Dye C, Arrighi HM (2013) Rate of conversion from prodromal Alzheimer's disease to Alzheimer's dementia: A systematic review of the literature. Dement Geriatr Cogn Disord Extra 3, 320-332.

[4] Giau VV, Bagyinszky E, An SSA (2019) Potential fluid biomarkers for the diagnosis of mild cognitive impairment. Int J Mol Sci 20, 4149.

[5] Cunningham C, Hennessy E (2015) Co-morbidity and systemic inflammation as drivers of cognitive decline: New experimental models adopting a broader paradigm in dementia research. Alzheimers Res Ther 7, 33.

[6] Krstic D, Madhusudan A, Doehner J, Vogel P, Notter T, Imhof C, Manalastas A, Hilfiker M, Pfister S, Schwerdel C, Riether C, Meyer U, Knuesel I (2012) Systemic immune challenges trigger and drive Alzheimer-like neuropathology in mice. J Neuroinflammation 9, 151.

[7] Pendlebury ST, Rothwell PM (2009) Prevalence, incidence, and factors associated with pre-stroke and post-stroke dementia: A systematic review and meta-analysis. Lancet Neurol 8, 1006-1018.

[8] Christensen JL, Tan S, Chung HE, Ghosalkar DS, Qureshi R, Chu A, Yu W, Berus J, Shah NR, Wu W-C, Chun H, Aikawa E, Choudhary G, Morrison AR (2020) Aortic valve calcification predicts all-cause mortality independent of coronary calcification and severe stenosis. Atherosclerosis 307, 16-20.

[9] Rodriguez CJ, Bartz TM, Longstreth WT, Jr., Kizer JR, Barasch E, Lloyd-Jones DM, Gottdiener JS (2011) Association of annular calcification and aortic valve sclerosis with brain findings on magnetic resonance imaging in community dwelling older adults: The cardiovascular health study. $J$ Am Coll Cardiol 57, 2172-2180.

[10] Hahn K, Myers N, Prigarin S, Rodenacker K, Kurz A, Forstl H, Zimmer C, Wohlschlager AM, Sorg C (2013) Selectively and progressively disrupted structural connectivity of functional brain networks in Alzheimer's disease - revealed 
by a novel framework to analyze edge distributions of networks detecting disruptions with strong statistical evidence. Neuroimage 81, 96-109.

[11] Knopman DS, Griswold ME, Lirette ST, Gottesman RF, Kantarci K, Sharrett AR, Jack CR, Jr., Graff-Radford J, Schneider AL, Windham BG, Coker LH, Albert MS, Mosley TH, Jr., Investigators AN (2015) Vascular imaging abnormalities and cognition: Mediation by cortical volume in nondemented individuals: Atherosclerosis risk in communities-neurocognitive study. Stroke 46, 433-440.

[12] Wolters FJ, Bos D, Vernooij MW, Franco OH, Heart-Brain Connection collaborative research g, Hofman A, Koudstaal PJ, van der Lugt A, Ikram MA (2017) Aortic valve calcification and the risk of dementia: A population-based study. J Alzheimers Dis 55, 893-897.

[13] Moyer VA, Force USPST (2014) Screening for lung cancer: U.S. Preventive Services Task Force recommendation statement. Ann Intern Med 160, 330-338.

[14] Agatston AS, Janowitz WR, Hildner FJ, Zusmer NR, Viamonte M, Jr., Detrano R (1990) Quantification of coronary artery calcium using ultrafast computed tomography. J Am Coll Cardiol 15, 827-832.

[15] Shavelle DM, Budoff MJ, Buljubasic N, Wu AH, Takasu J, Rosales J, Otto CM, Zhao XQ, O’Brien KD (2003) Usefulness of aortic valve calcium scores by electron beam computed tomography as a marker for aortic stenosis. Am J Cardiol 92, 349-353.

[16] Christensen JL, Sharma E, Gorvitovskaia AY, Watts JP, Jr., Assali M, Neverson J, Wu WC, Choudhary G, Morrison AR (2019) Impact of slice thickness on the predictive value of lung cancer screening computed tomography in the evaluation of coronary artery calcification. J Am Heart Assoc 8, e010110.

[17] Thygesen K, Alpert JS, Jaffe AS, Chaitman BR, Bax JJ, Morrow DA, White HD, Executive Group on behalf of the Joint European Society of Cardiology (ESC)/American College of Cardiology (ACC)/American Heart Association (AHA)/World Heart Federation (WHF) Task Force for the Universal Definition of Myocardial Infarction (2018) Fourth universal definition of myocardial infarction (2018). J Am Coll Cardiol 72, 2231-2264.

[18] Quan H, Li B, Saunders LD, Parsons GA, Nilsson CI, Alibhai A, Ghali WA, IMECCHI Investigators (2008) Assessing validity of ICD-9-CM and ICD-10 administrative data in recording clinical conditions in a unique dually coded database. Health Serv Res 43, 1424-1441.

[19] Nasreddine ZS, Phillips NA, Bã@Dirian VR, Charbonneau S, Whitehead V, Collin I, Cummings JL, Chertkow H (2005) The Montreal Cognitive Assessment, MoCA: A brief screening tool for mild cognitive impairment. $J \mathrm{Am}$ Geriatr Soc 53, 695-699.
[20] Tsoi KKF, Chan JYC, Hirai HW, Wong SYS, Kwok TCY (2015) Cognitive tests to detect dementia. JAMA Intern Med $\mathbf{1 7 5}, 1450$.

[21] (2016) Educational Attainment in the United States: 2015. Report Number P20-578. https://www.census. gov/library/publications/2016/demo/p20-578.html

[22] Clavel MA, Messika-Zeitoun D, Pibarot P, Aggarwal SR, Malouf J, Araoz PA, Michelena HI, Cueff C, Larose E, Capoulade R, Vahanian A, Enriquez-Sarano M (2013) The complex nature of discordant severe calcified aortic valve disease grading: New insights from combined Doppler echocardiographic and computed tomographic study. $J \mathrm{Am}$ Coll Cardiol 62, 2329-2338.

[23] Clavel MA, Pibarot P, Messika-Zeitoun D, Capoulade R, Malouf J, Aggarval S, Araoz PA, Michelena HI, Cueff C, Larose E, Miller JD, Vahanian A, Enriquez-Sarano M (2014) Impact of aortic valve calcification, as measured by MDCT, on survival in patients with aortic stenosis: Results of an international registry study. J Am Coll Cardiol 64, 1202-1213.

[24] Teo KK, Corsi DJ, Tam JW, Dumesnil JG, Chan KL (2011) Lipid lowering on progression of mild to moderate aortic stenosis: Meta-analysis of the randomized placebocontrolled clinical trials on 2344 patients. Can J Cardiol 27, 800-808.

[25] Carabello BA (2010) The SEAS Trial. Curr Cardiol Rep 12, 122-124.

[26] Cowell SJ, Newby DE, Prescott RJ, Bloomfield P, Reid J, Northridge DB, Boon NA (2005) A randomized trial of intensive lipid-lowering therapy in calcific aortic stenosis. N Engl J Med 352, 2389-2397.

[27] Chan KL, Teo K, Dumesnil JG, Ni A, Tam J, Investigators A (2010) Effect of Lipid lowering with rosuvastatin on progression of aortic stenosis: Results of the aortic stenosis progression observation: Measuring effects of rosuvastatin (ASTRONOMER) trial. Circulation 121, 306-314.

[28] de Torbal A, Boersma E, Kors JA, van Herpen G, Deckers JW, van der Kuip DA, Stricker BH, Hofman A, Witteman JC (2006) Incidence of recognized and unrecognized myocardial infarction in men and women aged 55 and older: The Rotterdam Study. Eur Heart J 27, 729-736.

[29] VanderWeele TJ, Ding P (2017) Sensitivity analysis in observational research: Introducing the E-value. Ann Intern Med 167, 268-274.

[30] Holder K (2008) The educational attainment of veterans: 2007. US Census Bureau. 